\title{
Introduction of a junior doctors' handbook: an essential guide for new doctors
}

\author{
Daniella Ross, Claire Petrie, Vicki Tully
}

NHS Tayside

\begin{abstract}
The transition period for new junior doctors is a daunting and challenging time, as vast amounts of information specific to each hospital, ward, and job must be learnt while maintaining patient care standards.[1] In NHS Tayside, Scotland, tips and guidance for each job are informally handed over from previous junior doctors to the next, resulting in an unreliable and unsustainable handover of information. Time must then be spent by new doctors learning the intricacies and practicalities of their new job, rather than spending time focusing on patient care.

Our aim was to improve this transition period for new junior doctors to NHS Tayside through the creation and implementation of a junior doctors' handbook, which would provide information and practical advice on day to day life as a junior doctor. We hoped to implement this project by August 2015 to coincide with the arrival of these new doctors to NHS Tayside. Through repeat PDSA cycles we created a sustainable and reliable junior doctors' handbook, containing a centralised hub of information for doctors that was accessible through our health board's website. The junior doctors' handbook has been a highly beneficial resource that has been praised for its detailed information on all aspects of day to day life for doctors in NHS Tayside. Feedback also demonstrated that doctors felt the junior doctors' handbook had improved their efficiency. Our hope is that this project can continue to be developed within our hospital, but also to be used as an idea outside our health board to improve the transition period for new doctors on a wider scale.
\end{abstract}

\section{Problem}

Each year in August, hospitals throughout the UK see the arrival of recently graduated new junior doctors arriving on the wards. They must almost instantly slip into the position of their predecessors by becoming familiar with their role, the job, and the ward itself. This transition period is well known to be a challenging and daunting time for new doctors, as vast amounts of information specific to each hospital, ward, and job must be learnt while patient care standards are maintained.[1]

Prior to even stepping foot onto a ward, new doctors must first familiarise themselves with their new environment: the practicalities of finding their new hospital, using staff car parks, looking up bus routes, and locating their ward. Their task is then to become familiar with the new ward itself: the daily routines of ward rounds, the introduction to all team members, the location of equipment, and then the use of computer programs to request and review investigations. Alongside this comes the challenge of managing unwell patients, prescribing medications, sourcing guidelines or senior help, finding contact numbers for different departments, admitting and discharging patients, contacting rota organisers to request annual leave, as well as the completion of mandatory requirements for portfolio development.

In NHS Tayside, Scotland, there is a shadowing period for new foundation year (FY) doctors, and an introductory period for other junior doctors prior to starting work. This allows the transfer of more general information from a variety of staff, an overview to working in the hospital and of the IT services, as well as an opportunity to spend time on the ward familiarising themselves with their new role and environment. Information specific to the working life of a junior doctor in NHS Tayside, alongside tips and guidance for each new job, is informally passed to new junior doctors through word of mouth from outgoing doctors. This unfortunately results in an unreliable and unsustainable handover allowing for key items of information to be missed, which ultimately leads to the compromise of patient care.[2]

Our aim was to improve the transition period for new junior doctors to NHS Tayside through the creation and implementation of a junior doctors' handbook by August 2015, to coincide with the arrival of new junior doctors. The junior doctors' handbook would provide information and practical advice on day to day life as a junior doctor working in NHS Tayside.

\section{Background}

Since 2007 and the introduction of Modernising Medical Careers,[3] the majority of doctors in training change rotation during the first week of August. "Black Wednesday," as it has been unfortunately named, sees an increase in patient morbidity and mortality.[4] Communication within medicine is paramount, and an effective handover of information is essential to maintain high standards of patient care during this transition period.[1, 2, 5] New FY doctors receive an induction week prior to starting work, which contains teaching, advice from various healthcare professionals, and the shadowing of previous FYs. Due to the vast amounts of information delivered, coupled with nerves and potential fears about starting work, this information can be difficult to retain.[5]

The idea of a handbook stemmed from the East of Scotland's 
BMJ Quality Improvement Reports

Junior Doctor's Forum as a project to improve the junior doctor induction period, and thereby improve patient care. Handbooks for new doctors are commonly used in other NHS trusts and are given out during induction weeks; however, printed information quickly becomes outdated and is then difficult to update.[5] Alongside this, the information provided is rarely produced by junior doctors, and therefore doesn't include integral information that can only be learnt from working on the wards.[5] This includes information regarding hospital layout, documentation, ordering investigations, making referrals, on call information, prescribing, guidelines, IT systems, and contact numbers.

By creating an easily accessible online handbook, written by junior doctors for junior doctors, the aim was to end the recurring problem of an unreliable handover of information to new doctors. Patient care could therefore be maintained during this transition period, as information regarding practical advice and information specific to our health board could be accessed through the handbook.

\section{Baseline measurement}

A retrospective study was undertaken using foundation year one (FY1) doctors working within NHS Tayside, who began their training prior to the implementation of the junior doctors' handbook. Baseline data was collected through an online questionnaire which was sent to the 93 doctors fulfilling these criteria, of which thirty completed the survey. The questionnaire asked questions regarding how prepared junior doctors felt on starting work, which areas they felt most unprepared with, and what information they would have wanted to know prior to starting work. The questionnaire also allowed for free text feedback on what junior doctors felt would be useful to have in a handbook.

Twelve of the $30 \mathrm{FY} 1 \mathrm{~s}(40 \%)$ completing the survey felt they were unprepared on starting work, despite their induction week. Eight FY1s (26\%) felt out of their depth on a daily basis when starting their new jobs. The majority of doctors felt prepared with regards to management of acutely unwell patients, clinical knowledge, prescribing, and practical procedures. However, they felt they were unprepared in utilising computer programs, performing administrative tasks, handing over information at formalised handover, and making referrals.

Nearly all participants (29 out of 30 respondents) felt that a handbook containing key information for their new job would have eased their transition period for starting work, and would be a useful resource to continue to use throughout the year. The most useful information highlighted from the survey to be included in a handbook, in order, was as follows:

\section{- Useful contact numbers}

- Information on hospital at night and handover

- Information on utilising computer programs specific to NHS Tayside

- Making referrals
- E-portfolio.

Free text feedback highlighted the need for the handbook to be easily accessible, preferably as an online handbook, and for it to contain information specific to NHS Tayside that was written by junior doctors, and therefore relevant and reliable.

\section{Design}

Our intervention took the form of a junior doctors' handbook created prior to the arrival of the new doctors in August 2015, which would be an online resource available on both computers and smartphones. The purpose of the handbook was to have an easily accessible, centralised hub of information that would contain relevant information for junior doctors in a clear and concise format, so that it could be utilised by new doctors starting work within NHS Tayside.

The information contained within the handbook aimed to be divided into four main sections, as highlighted by our feedback from our initial questionnaire. These sections were day to day life on the wards, information on NHS Tayside and its hospitals, how to access guidelines, and a list of useful contact numbers. The site was designed to be easily accessible and sustainable, to have information in a clear and concise format and layout, as well as also being user focused and user friendly.

Information was to be collated for the handbook through personal experience as well as through feedback from a variety of relevant senior clinicians, and through feedback from junior doctors via a focus group. The concept of the handbook is highly sustainable, as after setting up the site there are limited maintenance costs, and the site can continue to be used and updated as appropriate. The website was initially launched on external website platform Wix (www.wix.com); however, we hoped to incorporate the junior doctors' handbook into the NHS Tayside website, therefore allowing for an easily updateable resource for years to come. With approval through IT services the sites were incorporated in August 2015, and the handbook could therefore be more easily accessed by users, and could also be updated with amendments through IT services.

Overall, we hoped to design and create a user friendly website that was easily accessible and updateable, that would be sustainable, reliable, and would continue to be used by NHS Tayside for years to come.

\section{Strategy}

A total of three PDSA cycles were conducted, and more detailed evidence of these can be found in appendix 1 .

PDSA cycle 1

We surveyed junior doctors prior to the implementation of the handbook to establish what information they wished they had known prior to starting work, and what information they would like to see included in a handbook. Ninety seven percent ( 29 out of 30 
respondents) felt a handbook would have been useful, and wanted to see information and practicalities of working as a junior doctor in NHS Tayside included. We aimed to therefore create the first version of the junior doctors' handbook through external website platform Wix before gaining feedback on its use.

PDSA cycle 2

We gained formal feedback on the content, usability, accessibility, and layout of the junior doctors' handbook after its creation on the external website, through a junior doctor focus group and feedback from senior clinicians. Feedback regarding content was highly complimentary, and numerous suggestions for development were made. A noted criticism was the poor usability of the site on NHS Tayside's intranet. We planned to incorporate the junior doctors' handbook into NHS Tayside's intranet, and amend content as highlighted through feedback prior to the launch of the site in August 2015.

PDSA cycle 3

We formally evaluated the junior doctors' handbook after its incorporation to the NHS Tayside site and its launch in August 2015. We gained feedback on how it had eased the transition for new doctors to NHS Tayside, as well as feedback on user satisfaction, site layout, accessibility, sustainability, and usability. We also collated usage data on the site. Feedback demonstrated the site had been accessed 1055 times during August 2015, that FY doctors felt it had improved their efficiency on the wards, and that it was a highly beneficial resource. The junior doctors' handbook was praised on its sustainability, usability, layout, and accessibility. Challenges with access to the site on smartphones were highlighted, as well as difficulty in accessing the guidelines offline. Feedback from users demonstrates the junior doctors' handbook has been a highly successful intervention, and it has vast scope to be expanded within and outside of NHS Tayside. Our future aim is to improve accessibility through smartphones via the creation of an app, and to develop this resource to being specialty specific.

See supplementary file: ds6598.docx - "Appendix 1. PDSA cycles junior doctors' handbook"

\section{Post-measurement}

We designed, created, and implemented the junior doctors' handbook, which can be accessed at www.nhstjuniordocshandbook.scot.nhs.uk. Post intervention measurement was comprised initially of the collection of usage data from the site, before a survey was sent to new FY1 doctors from the August 2015 intake, gaining feedback specifically on user satisfaction, usability, accessibility, layout, and sustainability.

Usage data was collected for the month of August 2015, which showed that the junior doctors' handbook had been accessed 1055 times. The majority (831 out of 1055) of these visits had been on the "Day to Day" subheading of the site, which includes practical information for day to day life of the wards, including information on handover, ward rounds, discharging patients, e-portfolio, and the utilisation of computer systems.

Alongside this, post intervention measurement took the form of a survey which was sent to all FY1 doctors from the August 2015 intake, after the junior doctors' handbook had been launched during their induction week. Twenty three out of $96 \mathrm{FY} 1$ doctors completed the survey. Twenty two out of 23 respondents (96\%) felt the junior doctors' handbook was beneficial to have on starting their new job, and the majority ( 16 out of $23 ; 70 \%$ ) stated that it improved their efficiency as a doctor. Twenty two percent of respondents stated they used the handbook on a daily basis, and $70 \%$ used it on a weekly basis. Ninety six percent found the handbook easily accessible from a computer, however only $55 \%$ found it accessible from a smartphone. Free text feedback highlighted that this was as due to the site being inaccessible without internet.

Data relating to user satisfaction was collated through qualitative feedback from the survey sent to junior doctors. There were many positive comments given, and this data demonstrated evidence of self reported improvements after utilising the junior doctors' handbook. Qualitative feedback was as follows:

- "Invaluable resource for starting work in a trust where I had never worked previously. It answered every possible question I could have had. I would have been lost without it."

- "Very helpful and relevant information, very grateful to have had it as it made the first few days of work so much better."

- "Lots of good information I wished l'd known sooner."

- "I am utterly shocked at how the online handbook covers every situation I could imagine could become a problem. I am gobsmacked at how extensive it is and it will be incredibly valuable."

There were a number of criticisms highlighted from our feedback; these were felt to not have been previously demonstrated, as the junior doctors surveyed in our post intervention measurement had not worked without the junior doctors' handbook in place. Previous feedback had been collated with the launch of the handbook, and hence was more positive. The main criticism with the junior doctors' handbook was its offline use on smartphones. A number of suggestions were made to create an app that could be downloaded and updated as appropriate, to prevent the need for Wi-Fi access. There were also many areas that were highlighted as potential areas for development; these included creating an area on the site for specific specialities, to improve the unreliable handover given between each four month job rotation; creating an area to "ask a junior doctor" questions; and also an area with more practical advice for starting work (eg with regards to team working, and challenging colleagues).

\section{Lessons and limitations}

Overall, there were limited setbacks to the project. There was good engagement from stakeholders, as it was clear this project was going to be an invaluable and sustainable resource that would benefit NHS Tayside for years to come. 
The main challenge for the junior doctors' handbook came with accessibility of the site. The site was initially inaccessible through NHS Tayside internet when hosted on the external website. The challenge came from the transition of moving the website from the external host on to the NHS Tayside website. The initial aim was to have the website based within the Tayside intranet "Staffnet" site, to therefore allow open access to the intranet guidelines also hosted within the Staffnet site; however, this unfortunately became too challenging and hence a decision was made for it to be hosted on NHS Tayside's external website. There was immense time pressure to have the handbook incorporated into the site prior to the arrival of the new junior doctors to NHS Tayside, and with excellent support from the Medical Education Directorate and the IT staff this was completed. The site in its current form hosted on NHS Tayside's external website has ongoing problems with accessibility to guidelines, as these are solely accessed through NHS Tayside's desktop computers or its Wi-Fi. Guidelines are therefore inaccessible through smartphones or personal computers, and hence we aim to create a smartphone application to allow secure access to these guidelines. Our eventual aim is to incorporate this handbook into the Staffnet site, in order to finally solve these accessibility issues.

Another challenge faced was the vast amount of work needed to create the content for the handbook, as this was taken from personal experiences working within NHS Tayside. This information was collated and then reviewed by more senior clinicians; it therefore relied heavily on feedback, which was a timely process. In hindsight it would have been easier to develop and collate information using a variety of both junior and senior staff, along with a wider quality improvement team to create and implement the project. The aim is to continue to develop the junior doctors' handbook by incorporating experiences from a variety of staff including senior clinicians, specialist nurses, and pharmacists, in order to create a resource that isn't solely limited to use by junior doctors, but that could be used for doctors of all levels of seniority.

This project was also our junior doctors' first quality improvement project, and hence there were a number of changes that in hindsight we would have implemented from the beginning. We hoped to improve junior doctor efficiency, allowing them to spend more time with patients rather than learning the intricacies and practicalities of the job. We have received extremely positive qualitative feedback, however we have limited quantative feedback on the impact of the handbook. In retrospect we would have improved our quantative measures through calculation of the time saved for junior doctors through the creation of our handbook, as well as measures relating to improvements in patient safety.

We had also underestimated the impact this project would have within NHS Tayside. Initially we had aimed to create this solely for foundation year doctors, however this was quickly expanded to include all junior doctors. It has been used, and continues to be used by junior doctors of all levels of seniority after its launch in August 2015, during their transition periods prior to starting work. Teaching sessions were delivered on the site and its use at both of these inductions. As well as this, the site has been introduced into the fourth and fifth year medical student curriculum at the University of Dundee.

Feedback highlights that the junior doctors' handbook is continuing to be used by the majority of junior doctors on a minimum of a weekly basis; with support from NHS Tayside it is hoped that we can continue to expand and develop this invaluable resource. With regards to sustainability of the junior doctors' handbook, in the short term we plan to continue to lead this project to address the issues raised regarding accessibility and usability offline, by developing this resource into a smartphone app as earlier discussed. We also hope to develop the handbook into being specific for each job rotation, to further expand the use of this resource. In the long term we plan to see our team expanded through the incorporation of more junior doctors; however, the ultimate responsibility for the maintenance, development, and upkeep of the junior doctors' handbook will be taken by the East of Scotland Junior Doctor's Forum, alongside support from NHS Tayside's Quality Assurance Officer and IT departments. There is vast scope for further development of the junior doctors' handbook within NHS Tayside, and this format also has the potential to be implemented further afield to other hospitals outside our health board.

\section{Conclusion}

Our aim was to improve the August transition period for new junior doctors to NHS Tayside through creation and implementation of a junior doctors' handbook, which would provide information and practical advice on day to day life as a junior doctor. Through quality improvement and a number of PDSA cycles, we have created an easily accessible, sustainable, reliablem, and updateable resource that most importantly has excellent user satisfaction. This resource has undoubtedly been a success and has potentially improved the efficiency of junior doctors, allowing them to spend more time on patient care rather than learning and relearning the intricacies and practicalities of their new job from scratch. There was a clear need for there to be a centralised hub of information, and the junior doctors' handbook has become an invaluable resource for the new junior doctors in NHS Tayside. There is vast scope for this project to be expanded, and we hope to see this resource developed within and outside our health board in the future.

\section{References}

1. General Medical Council. Promoting excellence: standards for medical education and training. http://www.gmcuk.org/education/standards.asp

2. British Medical Association. Safe handover: safe patients. Guidance on clinical handover for clinicians and managers. London: British Medical Association, 2004.

3. Modernising Medical Careers. www.mmc.nhs.uk (accessed August 2015)

4. Jen MH, Bottle A, Majeed A, Bell D, Aylin P. Early inhospital mortality following trainee doctors' first day at work. PLoS One 2009;4(9):e7103.

5. Davies M, Panchal S, Misra N. The Handbook: an end to 'I wish I had known that before I started'. BMJ Qual Improv 


\section{BMJ Quality Improvement Reports}

Report 2015;4: doi:10.1136/bmjquality.u203210.w1579

\section{Declaration of interests}

We have read and understood BMJ policy on declaration of interests and declare that we have no competing interests.

\section{Acknowledgements}

Craig Butler, Jordan Napier, Karen Lambie, Dr Emily Ward,

Tayside's IT Services, Dr Neil McGuchan and the East of Scotland Junior Doctor's Forum, and all colleagues who provided feedback and completed questionnaires.

\section{Ethical approval}

The work being reported was deemed exempt from ethics review according to local policy. 